\title{
Adaptive Management of the Moment of Radiation of Laser Energy at Suppression Optical-Electrical Means
}

\author{
Alexandr N. Glushkov, \\ Juriy L. Koziratsky and Ruslan E. Merkulov* \\ Military Education and Research Centre of Military-Air Forces \\ «Military-Air Academy \\ Named After Professor N.E. Zhukovsky and Yu.A. Gagarin» \\ 54a Starykh Bolshevikov Str., Voronezh, 394064, Russia
}

The algorithm of management is developed by the moment of radiation of laser hindrances at suppression optical-electrical means of looking type. Decision-making on "shot" is made with use of criterion of Neumann-Pirson's on the basis of the forecast of size of capacity of a laser hindrance for entrance pupil optical-electrical means and probability of excess by it of a threshold of suppression optical-electrical means for the period of not less set.

Keywords: laser hindrances, functional defeat, probability of acceptance of the correct decision on a shot, probability of acceptance of the erroneous decision on a shot.

Citation: Glushkov A.N., Koziratsky Ju.L., Merkulov R.E. Adaptive management of the moment of radiation of laser energy at suppression optical-electrical means, J. Sib. Fed. Univ. Eng. technol., 2019, 12(1), 81-87. DOI: 10.17516/1999-494X-0031.

(C) Siberian Federal University. All rights reserved

This work is licensed under a Creative Commons Attribution-NonCommercial 4.0 International License (CC BY-NC 4.0).

* Corresponding author E-mail address: rus_212@mail.ru, urleo@bk.ru 


\title{
Адаптивное управление моментом излучения лазерной энергии \\ при подавлении оптико-электронных средств
}

\author{
А.Н. Глушков, Ю.Л. Козирацкий, Р.Е. Меркулов \\ Военный учебно-научный изентр Военно-воздушных сил \\ «Военно-воздушная академия \\ имени профессора Н.Е. Жуковского и Ю.А. Гагарина» \\ Россия, 394064, Воронеж, ул. Старых Большевиков, 54 а
}

Разработан алгоритм управления моментом излучения лазерных помех при подавлении оптико-электронных средств смотрящего типа. Принятие решения на «выстрел» производится с использованием критерия Неймана-Пирсона на основе прогноза величины мощности лазерной помехи на входном зрачке ОЭС и вероятности превышения ею порога подавления оптико-электронных средств на время не менее заданного.

Ключевые слова: лазерные помехи, функциональное поражение, вероятность принятия правильного решения на выстрел, вероятность принятия ошибочного решения на выстрел.

\section{Введение}

При подавлении оптико-электронных средств (ОЭС) энергией лазерных помех остро стоит вопрос управления моментом их излучения. Это связано с необходимостью рационального расходования энергетического ресурса (боезапаса) лазерного комплекса. В настоящее время разработано достаточно большое количество способов управления моментом излучения лазерной энергии исходя из ослабления ее в атмосфере [1]. Данные способы позволяют прогнозировать статистические характеристики лазерного пучка в картинной плоскости цели. Вместе с тем они не позволяют прогнозировать вероятность подавления ОЭС в момент воздействия, что определяет высокую вероятность принятия ошибочных решений.

Цель работы состоит в разработке адаптивного алгоритма управления моментом излучения энергии лазерных помех при подавлении ОЭС исходя из обеспечения допустимого уровня ошибочных решений при производстве «выстрела».

\section{Постановка задачи}

Для формализации решаемой задачи и последующего получения решающего правила будем считать, что лазерный комплекс функционального подавления ОЭС включает в себя локационный канал (ЛК), помеховый канал (ПК) и подсистему управления (ПСУ). ЛК предназначен для поиска и обнаружения ОЭС, а также формирования целеуказания для наведения помехового излучения. В ПК формируется помеховое излучение с требуемыми характеристиками. В ПСУ реализуются алгоритмы управления работой комплекса. Далее представим условие подавления лазерной энергией ОЭС [2] в виде

$$
\begin{gathered}
E_{\text {nop }}^{-1} \int_{S_{o s c}} d^{2} \rho \int_{T_{s}} I(\vec{\rho}, t) d t \simeq \\
\simeq \eta_{\text {nop }} T_{D}^{-1} \tau_{+} \geq 1, \\
-82-
\end{gathered}
$$


где $S_{\text {оэс }}$ - площадь приемной апертуры ОЭС; $T_{D}$ - требуемая длительность воздействия на ОЭС лазерной энергией $E \geq E_{n o p} ; \eta_{n o p}=\frac{P_{o э c}}{P_{n o p}} ; P_{n o p}=\frac{E_{n o p}}{T_{D}} ; P_{о э c}, I(\vec{\rho}, t)$ мощность лазерных помех и распределение их интенсивности на входном зрачке ОЭС в момент воздействия; $\tau_{+}-$длительность

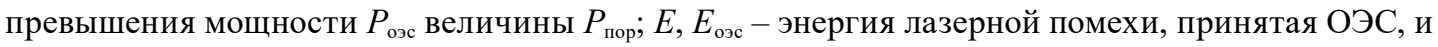
значение лазерной энергии, при котором произойдет поражение (подавление) ОЭС. Из (1) видно, что для принятия решения об излучении лазерных помех на ОЭС необходима информация о значениях $P_{\text {оэс }}$ и $\tau_{+}$. Данная информация может быть получена по результатам зондирования ОЭС локационным каналом комплекса.

\section{Решение}

В соответствии с [3] среднее значение принимаемого ЛК светового потока $P_{n 0}$ с точностью до постоянного множителя совпадает со средним потоком зондирующего излучения ЛК, принятым ОЭС $\bar{P}_{\text {Лоэс }}$ :

$$
\bar{P}_{n 0}=\bar{P}_{\text {Лоэс }} T_{a} \vartheta_{0} \cdot \frac{S_{n}}{L^{2}},
$$

где $T_{a}, \vartheta_{0}-$ коэффициенты, характеризующие прозрачность атмосферы и световозвращение ОЭС соответственно; $S_{n}$ - площадь приемной апертуры ЛК; $L$ - дальность до ОЭС. Выражение (2) позволяет по измеренным значениям мощности отраженного от ОЭС сигнала ЛК прогнозировать мощность излучения лазерных помех на входном зрачке ОЭС.

$$
\bar{P}_{\text {оэс }}=\frac{\bar{P}_{n o} L^{2}}{S_{n} T_{a} \vartheta_{0}} \cdot \frac{I_{n}}{I_{n}} \frac{Y_{n}^{2}}{Y_{n}^{2}}
$$

где $I, Y$ - плотность мощности на оси пучка и расходимость излучения лазера (индекс $\pi$ - соответствует локационному, а индекс $n$ - помеховому каналам). В соответствии с (1) решение на излучение лазерных помех должно быть принято на основе анализа величины отражённого ОЭС потока зондирующего пучка локационного канала при выполнении условия

$$
\eta_{\text {nop }} \tau_{+} \geq T_{D}=\frac{2 L}{c}+\tau_{0}+\tau_{B}
$$

где $c$ - скорость света; $\tau_{0}, \tau_{B}$ - время обработки принимаемого локационного сигнала и длительность воздействия на ОЭС излучением помех соответственно. В силу случайного характера принимаемого ЛК отраженного сигнала упомянутое решение может быть принято при двух взаимно исключающих условиях:

условие $\mathrm{A}_{1}$ - энергия излучения помех на ОЭС будет не ниже порогового уровня;

условие $\mathrm{A}_{0}$ - энергия излучения помех на ОЭС будет ниже порогового уровня.

Данным условиям могут соответствовать два вида решений:

решение $A_{1}^{*}$ - «выстрел» (излучение энергии помех);

решение $A_{0}^{*}$ - «не выстрел». 
Третьего решения («не знаю») быть не должно. Качественными показателями данных решений являются следующие условные вероятности: $P_{n \varepsilon}=P\left(A_{1}^{*} \mid A_{1}\right)$ - вероятность правильного «выстрела»; $P_{л в}=P\left(A_{1}^{*} \mid A_{0}\right)$ - вероятность ложного «выстрела»; $P_{n p}=P\left(A_{0}^{*} \mid A_{1}\right)$ - вероятность пропуска ОЭС; $P_{н в}=P\left(A_{0}^{*} \mid A_{0}\right)$ - вероятность правильного не выстрела, $P_{n 6}+P_{n p}=1$; $P_{л в}+P_{н \varepsilon}=1$. Вероятности $P_{л в}$ и $P_{n p}$ характеризуют возможность принятия ПСУ комплекса ошибочных решений, которые наказываются платой $r_{l 6}$ и $r_{n p}$ соответственно. В пределах зоны функционального поражения комплекса (область пространства, в пределах которой обеспечивается выполнение условия $P_{\text {оэс }} \geq P_{n o p}$ ) должны обеспечиваться требования: $P_{л \varepsilon} \leq P_{л ь}^{\text {до }}$ и $P_{n p} \leq P_{n p}^{\text {доn }}\left(P_{\pi в}^{\text {доn }}, P_{n p}^{\text {доn }}\right.$ - допустимые значения вероятностей принятия ошибочных решений). По аналогии с [4] при принятии решения на излучение помех необходимо минимизировать плату за ошибки, которая может быть охарактеризована средним риском, вычисляемым по формуле [4] $\bar{r}=r_{л 6} P_{л 6} P\left(A_{0}\right)+r_{n p} P_{n p} P\left(A_{1}\right)$. При равенстве $r_{n 6}=r_{n p}$ она принимает вид $\bar{r}=r_{n p} P\left(A_{1}\right)\left[P_{n p}+l_{0} P_{л 6}\right]$. Учитывая, что величина $r_{n p} P\left(A_{1}\right) \geq 0$, решающее правило по определению момента излучения лазерной энергии должно обеспечить минимизацию сомножителя $\left[P_{n p}+l_{0} P_{\text {лв }}\right]=\min$, в котором $l_{0}=P\left(A_{0}\right) / P\left(A_{1}\right)$. Такой подход соответствует критерию «идеального наблюдателя» [4], который целесообразно использовать при сравнении лазерных комплексов, а также при их оптимизации. В процессе боевого функционирования лазерных средств для принятия решения на выстрел более удобным является критерий Неймана - Пирсона [4], который позволяет обеспечить допустимые вероятности ложного «выстрела» и пропуска ОЭС $P_{n \varepsilon} \geq P_{n \varepsilon}^{m p}$ и $P_{л \varepsilon}<P_{л ь}^{\text {доn }}$. В качестве оценок вероятностей правильных и ошибочных решений на излучение лазерных помех примем следующие вероятности:

$$
\begin{aligned}
& \hat{P}_{n в}=P\left(P_{о э c} \geq P_{n o p}\right) P\left(\tau_{+} \geq T_{D}\right) ; \\
& \hat{P}_{л в}=P\left(P_{о э c} \geq P_{n o p}\right) P\left(\tau_{+}<T_{D}\right) ; \\
& \hat{P}_{n p}=\left(1-P\left(P_{о э c} \geq P_{n o p}\right) P\left(\tau_{+} \geq T_{D}\right)\right) ; \\
& \hat{P}_{\text {нв}}=\left(1-P\left(P_{\text {оэс }} \geq P_{n о р}\right) P\left(\tau_{+}<T_{D}\right)\right),
\end{aligned}
$$

где $P(\ldots)$ - вероятность выполнения соответствующего условия. Из (5) следует, что для определения момента излучения лазерных помех необходимо получение числовых значений вероятностей $P\left(P_{\text {оэс }} \geq P_{\text {пор }}\right), P\left(\tau_{+} \geq T_{D}\right)$ и $P\left(\tau_{+}<T_{D}\right)$. Решение на «выстрел» может быть принято на основе оценок значений $\hat{P}_{n \varepsilon}$ и $\hat{P}_{л ь}$ при выполнении условий:

$$
\hat{P}_{n \varepsilon} \geq P_{n \varepsilon}^{m p} \quad \text { u } \hat{P}_{л в}<P_{л в}^{\partial о n} .
$$

В выражении (6) $P_{n s}^{m p}$ и $P_{л ь}^{\text {до }}$ - заданная вероятность правильного выстрела и допустимая вероятность ложного выстрела соответственно. Для нахождения оценок $\hat{P}_{n}$ и $\hat{P}_{л 6}$ будем исходить из следующего. Принимаемый ОЭС поток лазерного излучения определяется суммой квадратов напряженностей поля помех в области пространства, которое опирается на плоскость ограниченную апертурой ОЭС. При нормальном законе распределения флуктуаций напряженности поля закон распределения флуктуаций светового потока определяется $\chi^{2}$ распределением с $n$ степенями свободы [5] (в рассматриваемом случае $n$ количество аддитивных компонент квадратов напряженности поля, определяющих величину светового потока). При $n \rightarrow \infty$ данное распределение аппроксимируется законом Гаусса и мы можем записать сле- 
дующее выражение для оценки вероятности выполнения условия превышения мощности $P_{\text {оэс }}$ величины $P_{\text {nор }}$ :

$$
\hat{P}\left(P_{\text {оэс }} \geq P_{\text {пор }}\right)=\Phi\left(\eta_{\text {nоp }}\right)=\frac{\sigma_{P}^{-1}}{\sqrt{2 \pi}} \int_{\eta_{\text {nop }}}^{\infty} e^{-\frac{\bar{P}_{o x c}^{2}}{2 \sigma_{P}^{2}(1-\eta)^{2}}} d \eta .
$$

В нем $\bar{P}_{о э с}=\left\langle P_{n o}\right\rangle \frac{L^{2}}{S_{n} T_{a} \vartheta_{0}} \cdot \frac{I_{n}}{I_{n}} \cdot \frac{Y_{n}^{2}}{Y_{n}^{2}} ; \sigma_{p}^{2}-$ оценка дисперсии флуктуаций мощности излучения лазерных помех на входном зрачке ОЭС.

$$
\sigma_{p}^{2} \simeq \sigma_{\pi}^{2}-\sigma_{\pi o}^{2}+\sigma_{p o}^{2},
$$

где $\sigma_{\pi}^{2} ; \sigma_{\text {ло }}^{2}$ - дисперсия флуктуаций мощности отраженного и зондирующего сигнала; $\sigma_{p o}^{2}-$ дисперсия флуктуаций мощности излучения помехового лазера.

Для оценки значений $P\left(\tau_{+} \geq T_{D}\right)$ и $P\left(\tau_{+}<T_{D}\right)$ воспользуемся результатами исследований законов распределения интервалов пересечений параметром Штреля порогового уровня [6]. Опираясь на данные исследования, выделим три случая: 1) $\bar{P}_{\text {оэс }} \gg P_{n o p}$; 2) $\bar{P}_{\text {оэс }} \sim P_{n o p}$;

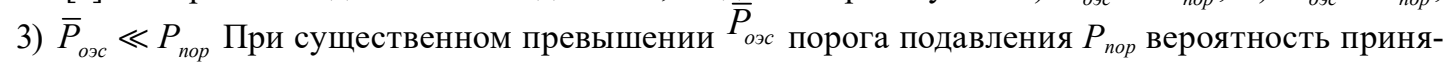
тия правильного решения на «выстрел», а также вероятность «ложного выстрела» могут быть оценены по следующим формулам:

$$
\begin{aligned}
& \hat{P}_{n в}=P\left(P_{\text {оэс }} \geq P_{n о р}\right) \exp \left(-T_{D} / \bar{\tau}_{+}\right), \\
& \hat{P}_{л в}=P\left(P_{\text {оэс }} \geq P_{n о р}\right)\left(1-\exp \left(-T_{D} / \bar{\tau}_{+}\right)\right),
\end{aligned}
$$

которые показывают, что оценивание $\hat{P}_{n \varepsilon}$ и $\hat{P}_{л в}$ требует сведений о значении $\bar{\tau}_{+}$. Эти сведения могут быть получены исходя из теории выбросов случайных процессов [4]. По аналогии с [6, 7] выражения для $P\left(P_{\text {оэс }} \geq P_{n о p}\right)$ и величины $\bar{\tau}_{+}$имеют следующий вид:

$$
\begin{aligned}
& \bar{\tau}_{+}=\frac{2 \pi}{\sqrt{-R_{0}^{\prime \prime}}} \Phi\left(\eta_{\text {nор }}\right) e^{\gamma^{2} / 2} ; \gamma=\frac{\bar{P}_{o э c}\left(1-\eta_{\text {nор }}\right)}{\sigma_{p}} ; \\
& \eta=\frac{P}{\bar{P}_{\text {oэc }}} ; R_{0}^{\prime \prime}=\left.\frac{d^{2} R(\tau)}{d \tau^{2}}\right|_{\tau=0} ; R(\tau)=R\left(t_{1}, t_{2}\right) .
\end{aligned}
$$

Во втором $\left(\bar{P}_{\text {оэс }} \sim P_{n о p}\right)$ и третьем $\left(\bar{P}_{\text {оэс }} \ll P_{n о р}\right)$ случаях законы распределения интервалов превышений световым потоком порогового уровня подчиняются логарифмически нормальному закону распределения и обобщенному закону Релея [6], которые при $\sigma_{P} / \bar{P}_{\text {оэс }} \ll 1$ аппроксимируются нормальным распределением. На основании этого вероятность $P\left(\tau_{+} \geq T_{D}\right)$ определяется выражением

$$
P\left(\tau_{+} \geq T_{D}\right)=1-F\left(\frac{T_{D}-\bar{\tau}_{+}}{\sigma_{+}}\right), F\left(T_{D}\right)=\frac{1}{\sigma_{+} \sqrt{2 \pi}} \int_{0}^{T_{D}} e^{-t^{2} / 2} d t ; t=\frac{T_{D}-\bar{\tau}_{+}}{\sigma_{+}},
$$

где $\sigma_{+}^{2}$-дисперсия интервалов превышения мощности $P_{\text {оэс }}$ величины $P_{n o p}$. При получении оценок по формуле (12) принимается, что при $\sigma_{P} / \bar{P}_{\text {оэс }} \ll 1$ отношение $\frac{\sigma_{+}}{\bar{\tau}_{+}} \approx \frac{\sigma_{P}}{\bar{P}_{\text {ээс }}}$.

$$
-85-
$$




\section{Заключение}

Таким образом, проведенные исследования позволяют сформулировать такой алгоритм определения момента излучения энергии лазерных помех. ОЭС облучается зондирующим сигналом ЛК лазерного комплекса. По отраженному сигналу делается прогноз параметров помехового излучения в плоскости ОЭС: $\bar{P}_{\text {оэс }}, \sigma_{P}^{2}, P\left(P_{\text {оэс }} \geq P_{n о р}\right)$ и $\bar{\tau}_{+}$. Сравниваются значения $\bar{P}_{\text {оэс }}$ и $P_{n o p}$. Оценивается вероятность превышения принимаемой ОЭС мощности помех порогового значения по формуле $P\left(\tau_{+} \geq T_{D}\right)=\exp \left(-T_{D} / \bar{\tau}_{+}\right)$при $P_{\text {оэс }} \gg P_{\text {пор }}$ или по формуле (12) в противном случае. По формулам (9) и (10) оцениваются вероятности принятия правильного и ошибочного решений на выстрел, который производится при выполнении условий (6).

Использование данного алгоритма позволит излучать лазерные помехи только при гарантии подавления ОЭС с заданной вероятностью и, как следствие, рационально расходовать энергетический ресурс лазерного комплекса.

\section{Список литературы}

[1] Лукин В.П. Атмосферная адаптивная оптика. Новосибирск, 1986. 172 с.[Lukin V.P. Atmospheric adaptive optics. Novosibirsk, 1986. 172 c.].

[2] Глушков А.Н., Козирацкий Ю.Л. Синтез лазерных помех координатно-метрическим оптико-электронным системам. Радиотехника, 2011, 8, 34-38.[Glushkov A.N., Koziratsky JU.L. Synthes of laser hindrances to koordinato-metric optiko-electronic systems, Radio engineering, 2011, 8, 34-38].

[3] Глушков А.Н., Кравцов Р.Н., Митрофанов А.Л. Модель локационного наблюдения ОЭС. Информационно-измерительные и управляющие системы, 2006, 7, [Glushkov A.N., Kravtsov R. $\mathrm{N}$, Mitrofanov A.L. Model lokacionnogo supervision OEC. Iinformation-measuring and operating systems, 2006, 7].

[4] Ширман Я.Д., Голиков В.И. Основы теории обнаружения радиолокационных сигналов и измерения их параметров. М.: Сов. радио, 1963, 278 с.[ Shirman J.D., Golikov V.I. Bases of the theory of detection of radar-tracking signals and measurement of their parametres. M.: Owls. Radio, 1963, 278 c.].

[5] Тихонов В.И. Нелинейные преобразования случайных процессов. М.:Радио и связь, 1986. 18. [Tikhonov V. I. Nonlinear transformations of casual processes. M.: Radio and communication, 1986. 185.].

[6] Глушков А.Н., Стрельцова Л.В. Статистические характеристики флуктуаций параметра Штреля относительно порогового уровня. Оптика атмосферы и океана. 1992, 2. [Glushkov A.N., Streltsova L.V. Statistical characteristics of fluctuations of parametre of Shtrelja concerning threshold level. Atmosphere and ocean Optics. 1992, 2].

[7] Глушков А.Н., Стрельцова Л.В. Начальные моменты выбросов параметра Штреля при адаптивной коррекции светового пучка. Оптический журнал, 1992, 3, 10-13 [Glushkov A.N., Streltsova L.V. Initial the moments of emissions of parametre of Shtrelja at adaptive correction of a light bunch. Optical magazine, 1992, 3, 10-13].

[8] Козирацкий Ю.Л. и др. Модели информационного конфликта средств поиска и обнаружения. М.: Радиотехника, 2013, 232. [Koziratsky J.L, etc. Models of the information conflict of means of search and detection. M.: Radio engineering, 2013. 232 c.].

$$
-86-
$$


[9] Козирацкий Ю.Л. и др. Модели пространственного и частотного поиска. М.: Радиотехника, 2014, 342 .[ Koziratsky J.L, etc. Models of spatial and frequency search. M: Radio engineering, 2014, 342 .].

[10]Козирацкий Ю.Л. Оптимизация угла расходимости излучения лазерной локационной системы в условиях помехи. Радиотехника, 1994, 3, 13-19 